\title{
Logic and social interaction: introduction
}

\author{
Sujata Ghosh • R. Ramanujam
}

Received: 28 July 2010 / Accepted: 28 July 2010 / Published online: 11 August 2010 (C) The Author(s) 2010. This article is published with open access at Springerlink.com

There is now a growing body of research on formal algorithmic models of social procedures and interactions between rational agents. These models attempt to identify logical elements within our day-to-day social activities. Largely using the language of logic and game theory, these studies have led to new insights into the dynamics of observation, updating of knowledge and belief, preference change, dialogues and processes of strategic interaction. Central to many of these studies is a multi-agent perspective on rational agency that situates inference in an interactive context. Logical studies of the role of communication, information exchange and even information hiding are broadening the scope of research on social interaction.

This special issue of Knowledge, Rationality and Action centres on the relation between epistemic attitudes and multi-agent interaction. We have five papers that explore different aspects of this relationship: how awareness determines knowledge and ignorance, how communications can ensure some agents' ignorance while increasing other agents' knowledge, what merging beliefs may mean in strategic terms, and what may be the linguistic desiderata for epistemic communication protocols. They typically employ models based on dynamic and temporal epistemic logics to study these questions. In all of them, one central issue contributes to complexity: intersubjectivity, whereby agents' reasoning about each others' awareness, knowledge and belief, is essential to the design and analysis of their interactions.

Agents' knowledge may in general be limited in many ways. One important way is by which they access such knowledge: agents observe events in the external world,

\footnotetext{
S. Ghosh

Department of Artificial Intelligence, University of Groningen, Groningen, The Netherlands e-mail: sujata@ai.rug.nl
}

R. Ramanujam ( $\varangle)$

The Institute of Mathematical Sciences, Chennai, India

e-mail: jam@imsc.res.in 
interact with other agents whereby they learn, and perform inferences. Access to knowledge may then be limited in some way by each of these mechanisms which determine what the agent is aware of. The paper by van Benthem and VelázquezQuesada studies awareness by splitting all information as those that come from external observations (and communication) and those obtained by inference. The dynamics of inference then becomes the focus of their study. The paper by Hoshi and Pacuit studies awareness and access by way of structural limitations which delimit what each agent is informed of. They then study the interaction between knowledge and (semi-private) communications under such awareness constraints.

The interdependence between agents' knowledge and the temporal evolution of system events is implicit in the studies mentioned above, but they are essential to security studies where what $A$ knows now about what $B$ did not know in the past can be critical. An important question then is to delimit the essential role played by epistemic operators in such information hiding situations, which is taken up by Dechesne and Wang. They survey approaches to security protocols modelled in epistemic logics.

Interaction between knowledge and communication in multi-agent scenarios is typically cast in a formal logic embedded in an informal communication language. However, logical studies demand formal semantics, and what results is often a somewhat superficial mix, that misses a great deal of operational detail, thereby raising questions about the value of the abstract analysis. On the other hand, if we consider programs instead of protocols, an elaborate theory of operational and denotational semantics exists for programming languages. This motivates van Eijck to propose goal extraction for epistemic protocols based on natural language semantics.

Much of the literature on epistemic interaction works with agents that operate individually. Yet, a major characteristic of social interaction is collective action based on epistemic attitudes shared by groups (to some degree). Pinning down such epistemics of groups is an important agenda of research, and Booth and Meyer take up one dimension of this, namely aggregating beliefs. When agents pool together their information and beliefs, some beliefs may have to be given up, leading to new equilibria, and characterizing these becomes their focus of study. The framework does not offer a specific logic but is based on abstract postulates that aggregation functions should conform to.

The special issue comes about as a response to the discussions held at the Workshop on Logic and Social Interaction that was held in the Institute of Mathematical Sciences, Chennai, India during 7-8 January, 2009, as a satellite event of the Third Indian Conference on Logic and Applications (Chennai, 9-11 January, 2009). We hope it exemplifies current logical approaches to modelling complex multi-agent interactive situations and provides a glimpse of the varied methodologies adopted for such modelling purposes.

We thank all the authors who submitted research articles for the special issue, and the reviewers for their detailed comments during the selection and revision processes. We thank Wiebe van der Hoek for encouragement and Ayrene Dialogo for editorial assistance. 
Open Access This article is distributed under the terms of the Creative Commons Attribution Noncommercial License which permits any noncommercial use, distribution, and reproduction in any medium, provided the original author(s) and source are credited. 